\title{
Longitudinal dispersion with constant source concentration along unsteady groundwater flow in finite aquifer: analytical solution with pulse type boundary condition
}

\author{
Mritunjay Kumar Singh ${ }^{1^{*}}$, Nav Kumar Mahato ${ }^{1}$, Premlata Singh ${ }^{2}$ \\ ${ }^{1}$ Department of Applied Mathematics, Indian School of Mines, Dhanbad, India; \\ *Corresponding Author: drmks29@rediffmail.com \\ ${ }^{2}$ Department of Mathematics, Banaras Hindu University, Varanasi, India; prema.singh@rediffmail.com
}

Received 24 December 2010; revised 25 January 2011, accepted 27 January 2011.

\begin{abstract}
Analytical solution is obtained to predict the contaminant concentration with presence and absence of pollution source in finite aquifer subject to constant point source concentration. A longitudinal dispersion along unsteady groundwater flow in homogeneous and finite aquifer is considered which is initially solute free that is, aquifer is supposed to be clean. The constant source concentration in intermediate portion of the aquifer system is considered with pulse type boundary condition and at the other end of the aquifer, concentration gradient is supposed to be zero. The Laplace Transformation Technique (LTT) is used to obtain the analytical solution of the formulated solute transport model with suitable initial and boundary conditions. The time varying velocities are considered. Analytical solutions are perhaps most useful for benchmarking the numerical codes and models. It may be used as the preliminary predictive tools for groundwater management.
\end{abstract}

Keywords: Aquifer; Unsteady Groundwater Flow; Longitudinal Dispersion; Uniform Source Concentration; Pulse Type Boundary Condition

\section{INTRODUCTION}

A large part of the drinking water in India comes from groundwater. The contamination of groundwater systems is still a major issue in the assessment of hazards and risks to public health. The underground systems are very attractive as waste repositories because of the possibility of degradation by biochemical processes. But in some cases, it can lead to contamination of regional groundwater systems. The contaminant releases to groundwater can occur by design, by accident or by negligence. Most of the groundwater contamination incidents involve substances released at or only slightly below the land surface. The transport of contaminants in groundwater is described by solute transport equations in the form of partial differential equations. These equations are known as solute transport models. These models simulate movement and concentration of various contaminants in groundwater system and can be classified into three categories such as advection models, advection-dispersion models, and advection-dispersion-chemical biological reaction models. Advection models define the movement of contaminant as a result of groundwater flow only. Advection dispersion models takes into consideration molecular diffusion, and microscopic/macro dispersion. Advection-dispersionchemical biological reacion models include the effect of chemical or biological reactions which change the concentration of transported contaminants [1-7].

The effects of initial and boundary conditions on the distribution of the tracer in time and distance for several one-dimensional systems (infinite, semi-infinite, and finite) were determined [8]. The effects of hydrodynamic dispersion, diffusion, radioactive decay, and simple chemical interactions of the tracer were included. An analytical method by which the effects of flow non uniformity and variable dispersion coefficients were evaluated for the problem involving longitudinal dispersion in porous media was proposed [9]. A boundary layer approximation was used to develop general solutions of the one-dimensional convective-dispersion equation for steady flow. Analytical solutions for two problems of longitudinal dispersion within semi-infinite, nonadsorbing, homogeneous, isotropic media in unidirectional flow fields were developed [10]. Dispersive sources in 
uniform groundwater flow were presented [11]. An analytical solution for the movement of a chemical in a porous medium as influenced by linear equilibrium adsorption, zero order production, and first order decay was presented [12]. An analytical solution for dispersion (in a finite non-adsorbing and adsorbing porous media) was developed [13,14] and it was controlled by flow (with unsteady unidirectional velocity distribution) of low concentration fluids towards a region of higher concentration. An analytical solution of the general one-dimensional solute transport model for confined aquifers was obtained [15]. An analytical solution for describing the transport of dissolved substances in heterogeneous porous media with a distance-dependent dispersion relationship was developed [16]. An analytical solution for the advection-dispersion equations with rate-limited desorption and first-order decay, using an Eigen function, integral equations method was derived [17]. Analytical solutions to two mathematical models for virus transport in one-dimensional homogeneous, saturated porous media for constant flux as well as constant concentration boundary conditions were presented [18]. The stochastic model for one-dimensional virus transport in homogeneous, saturated, semi-infinite porous media was developed [19]. The water table variation in response to time varying recharge was explored [20]. Analytical solutions to the transient, unsaturated transport of water and contaminants through horizontal porous media was presented [21]. Analytical solutions for sequentially coupled one-dimensional reactive transport problems were discussed [22]. Longitudinal dispersion with time dependent source concentration along unsteady groundwater flow in semi-infinite aquifer was presented [23]. Recently, one and two dimensional analytical solutions were also explored using Laplace and Hankel Transform Techniques respectively with suitable initial and boundary conditions [24,25].

In context of solute dispersion problem along unsteady groundwater flow, the objective of this study is to solve analytically convective-dispersive equation with an appropriate initial and boundary conditions. In the present work uniform source concentration in intermediate portion of the aquifer system is considered in splitting time domain i.e. the pulse type boundary condition which is not taken earlier [24]. The time dependent forms of velocities expressions are considered for numerical examples and discussion.

\section{ANALYTICAL SOLUTION FOR HOMOGENEOUS FINITE AQUIFER WITH PULSE TYPE BOUNDARY CONDITION}

Let $c(x, t)\left[\mathrm{ML}^{-3}\right]$ be the solute concentration at po- sition $x[\mathrm{~L}]$ at time $t[\mathrm{~T}]$ in homogeneous finite aquifer of length $\mathrm{L}$. Let $D\left[\mathrm{~L}^{2} \mathrm{~T}^{-1}\right]$ be the solute dispersion and $u\left[\mathrm{LT}^{-1}\right]$ be the velocity of the medium transporting the solute particles. Initially, aquifer is considered solute free i.e. aquifer is clean so the initial contaminant concentration is supposed to be zero at time $t=0$. Let $c_{0}$ $\left[\mathrm{ML}^{-3}\right]$ be the input contaminant concentration in intermediate portion of the aquifer system i.e. at $x=x_{0}$ till $t=t_{0}$ and beyond that it becomes zero. The contaminant concentration gradient at the other end of the aquifer i.e. at $x=L$ is supposed to be zero. The mathematical model for the contaminant concentration in space and time with pulse type boundary conditions can be written as follows:

$$
\begin{gathered}
D \partial^{2} c / \partial x^{2}-u \partial c / \partial x=\partial c / \partial t \\
u(t)=u_{0} V(t) \\
c(x, t)=0 ; x \geq x_{0}, t=0 \\
c(x, t)=\left\{\begin{array}{l}
c_{0} ; 0<t \leq t_{0}, x=x_{0} \\
0 ; t>t_{0}, x=x_{0}
\end{array}\right. \\
\partial c / \partial x=0 ; t \geq 0, x=L
\end{gathered}
$$

Here $u_{0}\left[\mathrm{LT}^{-1}\right]$ is the initial groundwater velocity at each $x$ and $V(t)$ is the time dependent expressions such as sinusoidally form i.e. $1-\sin m t$ and exponentially decreasing form i.e. $\exp (-m t), m t<1$ where $m$ $\left[\mathrm{T}^{-1}\right]$ is the flow resistance coefficients. The dispersion coefficient, vary approximately directly to seepage velocity for various types of porous media [26]. Also it was found that such relationship established for steady flow was also valid for unsteady flow with sinusoidal varying seepage velocity [27]. Let $D=a u$ where $a$ [L] is the dispersivity that can depend upon the pore size and geometry of porous medium. The physical system of the problem is represented by the Figure 1.

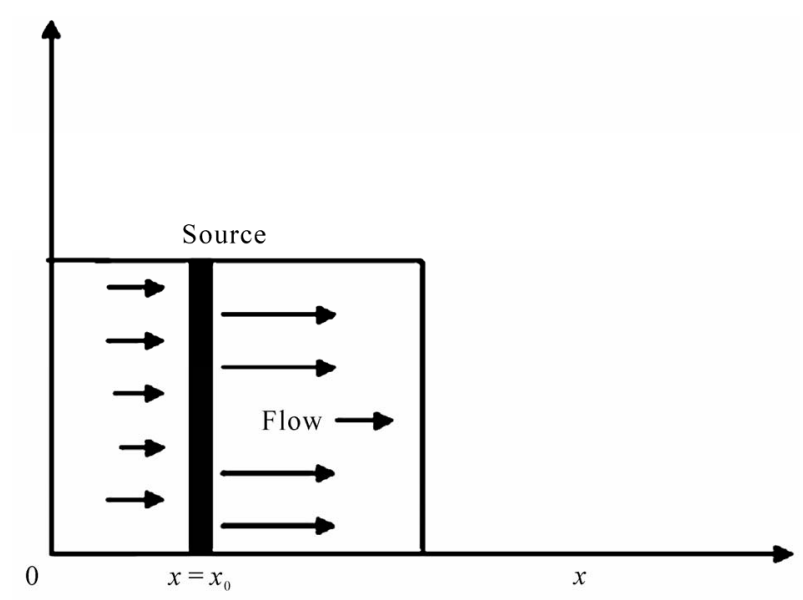

Figure 1. Physical system of the problem. 
Now using Eq.2, we get $D=D_{0} V(t)$. Here $D_{0}=a u_{0}$ is an initial dispersion coefficient.

Put $X=x-x_{0}$ and then Eqs.1-4 can now be written as

$$
\begin{gathered}
D_{0} \partial^{2} c / \partial x^{2}-u_{0} \partial c / \partial x=(1 / V(t)) \partial c / \partial t \\
c(X, t)=0 ; X \geq 0, t=0 \\
c(X, t)=\left\{\begin{array}{l}
c_{0} ; 0<t \leq t_{0}, X=0 \\
0, t>t_{0}, X=0
\end{array}\right. \\
\partial c / \partial X=0 ; T \geq 0, X=L-x_{0}
\end{gathered}
$$

A new time variable $T^{*}$, is introduced by the transformation [28]

$$
T^{*}=\int_{0}^{t} V(t) \mathrm{d} t
$$

and Eq.5 becomes

$$
D_{0} \partial^{2} c / \partial X^{2}-u_{0} \partial c / \partial X=\partial c / \partial T^{*}
$$

Now the set of non-dimensional variables are intro- duced as follows:

$$
Y=X / L, C=c / c_{0}, T=D_{0} T^{*} / L^{2}, U=u_{0} L / D_{0}
$$

The partial differential Eq.10 and corresponding initial and boundary conditions in non-dimensional form can be written as follows:

$$
\begin{gathered}
\partial^{2} c / \partial Y^{2}-U \partial C / \partial Y=\partial C / \partial T \\
C(Y, T)=0 ; Y \geq 0, T=0 \\
C(Y, T)=\left\{\begin{array}{l}
1 ; 0<T \leq T_{0}, Y=0 \\
0 ; T>T_{0}, Y=0
\end{array}\right. \\
\partial C / \partial Y=0 ; T \geq 0, Y=\left(L-x_{0}\right) / L
\end{gathered}
$$

Using the transformation

$$
C(Y, T)=K(Y, T) \exp \left(U Y / 2-U^{2} T / 4\right)
$$

in Eqs.12-15 and applying Laplace transformation, we can get the solution of obtained boundary value problem as follows:

$$
\begin{aligned}
& \bar{K}(Y, p)=\left[1 /\left(p-U^{2} / 4\right)\right]\left\{1-\exp \left[-\left(p-U^{2} / 4\right) T_{0}\right]\right\} \\
&\{\exp (-Y \sqrt{p})+[1-U /(\sqrt{p}+U / 2)][\exp (-(a-Y) \sqrt{p})-\exp (-(a+Y) \sqrt{p})] \\
&\left.-[1-U /(\sqrt{p}+U / 2)]^{2} \exp (-(2 a-Y) \sqrt{p})\right\} \\
& \bar{K}(Y, p)=\bar{K}_{1}(Y, p)+\bar{K}_{2}(Y, p)-\bar{K}_{3}(Y, p)
\end{aligned}
$$

where

$$
\begin{gathered}
\bar{K}_{1}(Y, p)=\left[1 /\left(p-U^{2} / 4\right)\right]\left\{1-\exp \left[-\left(p-U^{2} / 4\right) T_{0}\right]\right\} \exp (-Y \sqrt{p}) \\
\bar{K}_{2}(Y, p)=\left[1 /\left(p-U^{2} / 4\right)\right]\left\{1-\exp \left[-\left(p-U^{2} / 4\right) T_{0}\right]\right\}\{[1-U /(\sqrt{p}+U / 2)][\exp (-(a-Y) \sqrt{p})-\exp (-(a+Y) \sqrt{p})]\} \\
\bar{K}_{3}(Y, p)=\left[1 /\left(p-U^{2} / 4\right)\right]\left\{1-\exp \left[-\left(p-U^{2} / 4\right) T_{0}\right]\right\}\left\{[1-U /(\sqrt{p}+U / 2)]^{2}[\exp (-(2 a-Y) \sqrt{p})]\right\}
\end{gathered}
$$

Taking the inverse Laplace transform on (18), we get

$$
K(Y, T)=K_{1}(Y, T)+K_{2}(Y, T)-K_{3}(Y, T)
$$

where

$$
\begin{gathered}
K_{1}(Y, T)=\left\{\begin{array}{l}
F(Y, T) ; 0<T \leq T_{0} \\
F(Y, T)-F\left(Y, T-T_{0}\right) ; T>T_{0}
\end{array}\right. \\
K_{2}(Y, T)=\left\{\begin{array}{c}
{[F(a-Y, T)-F(a+Y, T)-U G(a-Y, T)+U G(a+Y, T)] ; 0<T \leq T_{0}} \\
{\left[F(a-Y, T)-F\left(a-Y, T-T_{0}\right)\right]-\left[F(a+Y, T)-F\left(a+Y, T-T_{0}\right)\right]} \\
-U\left[G(a-Y, T)-G\left(a-Y, T-T_{0}\right)\right]+U\left[G(a+Y, T)-G\left(a+Y, T-T_{0}\right)\right] ; T>T_{0}
\end{array}\right. \\
K_{3}(Y, T)=\left\{\begin{array}{c}
{\left[F(2 a-Y, T)-2 U G(a-Y, T)+U^{2} H(a-Y, T)\right] ; 0<T \leq T_{0}} \\
{\left[F(2 a-Y, T)-F\left(2 a-Y, T-T_{0}\right)\right]-2 U\left[G(2 a-Y, T)-G\left(2 a-Y, T-T_{0}\right)\right]} \\
+U^{2}\left[H(2 a-Y, T)-H\left(2 a-Y, T-T_{0}\right)\right] ; T>T_{0}
\end{array}\right.
\end{gathered}
$$


Finally, $K(Y, T)$ can be written as follows:

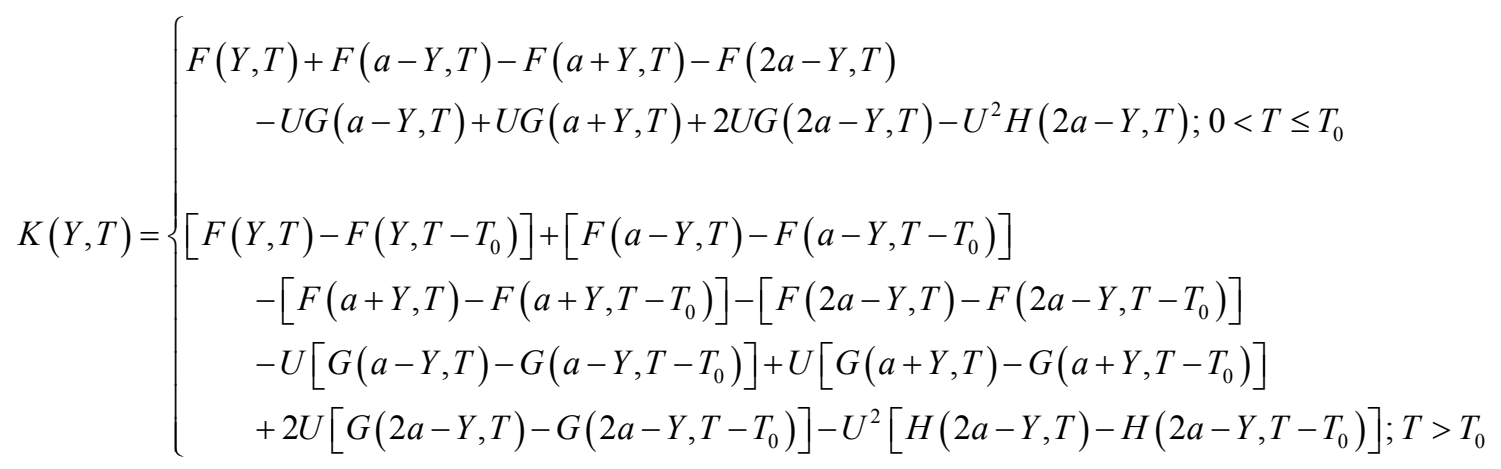

where

$$
\begin{gathered}
F(Y, T)=(1 / 2) \exp \left(U^{2} T / 4-U Y / 2\right) \operatorname{erfc}(Y / 2 \sqrt{T}-U \sqrt{T} / 2)+(1 / 2) \exp \left(U^{2} T / 4+U Y / 2\right) \operatorname{erfc}(Y / 2 \sqrt{T}+U \sqrt{T} / 2) \\
G(Y, T)=(\sqrt{T / \pi}) \exp \left(-Y^{2} / 4 T\right)+(1 / 2 U) \exp \left(U^{2} T / 4-U Y / 2\right) \operatorname{erfc}(Y / 2 \sqrt{T}-U \sqrt{T} / 2) \\
\quad-(1 / 2 U)\left(1+U Y+U^{2} T\right) \exp \left(U^{2} T / 4+U Y / 2\right) \operatorname{erfc}(Y / 2 \sqrt{T}+U \sqrt{T} / 2) \\
G(Y, T)=(-1 / U) \sqrt{T / \pi}\left(1+U Y / 2+U^{2} T / 2\right) \exp \left(-Y^{2} / 4 T\right) \\
+\left(1 / 2 U^{2}\right)\left(-1+U Y+2 U^{2} T+\left(U^{2} T / 2\right)(Y+U T)^{2}\right) \exp \left(U^{2} T / 4+U Y / 2\right) \operatorname{erfc}(Y / 2 \sqrt{T}+U \sqrt{T} / 2) \\
+\left(1 / 2 U^{2}\right) \exp \left(U^{2} T / 4-U Y / 2\right) \operatorname{erfc}(Y / 2 \sqrt{T}-U \sqrt{T} / 2)
\end{gathered}
$$

By substituting the values of $K(Y, T)$ in Eq.16 we may obtain the desired solution as

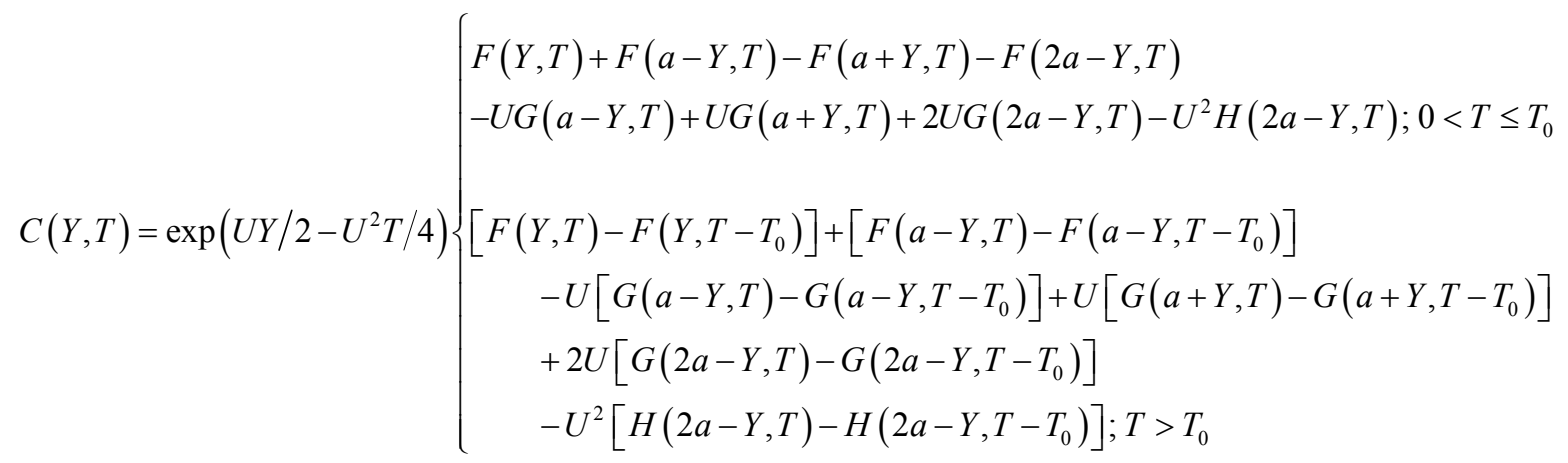

where $F(Y, T), G(Y, T)$ and $H(Y, T)$ are given in Equations (24)-(26).

\section{NUMERICAL EXAMPLE AND DISCUSSION}

Let us consider the sinusoidal and exponential forms of expressions are as follows:

$$
\begin{gathered}
V(t)=1-\sin m t \\
V(t)=\exp (-m t), m t<1
\end{gathered}
$$

where $m(/ \mathrm{d})$ is flow resistance coefficient. The exponential form of velocity expression was also considered to discuss dispersion in unsteady porous media flow [29]. For both the expressions, the non-dimensional time variable T may be written as

$$
\begin{gathered}
T=\left(D_{0} / m L^{2}\right)[m t-(1-\cos m t)] \\
T=\left(D_{0} / m L^{2}\right)[1-\exp (-m t)]
\end{gathered}
$$

where $m t=3 k+2$, where $k$ is the whole number. Here for $m=0.0165(/ \mathrm{d}),(28 \mathrm{a})$ yields, $t(\mathrm{~d})=182 k+121$ approximately. For these values of $m t$, the velocity $u$, is alternatively minimum and maximum. Hence it represents the groundwater level and velocity minimum during the month of June and maximum during December just after six months in one year. The next data of $t$ represents minimum and maximum records during June and December respectively in the subsequent years. These representations have been made in the Figure 2 . 


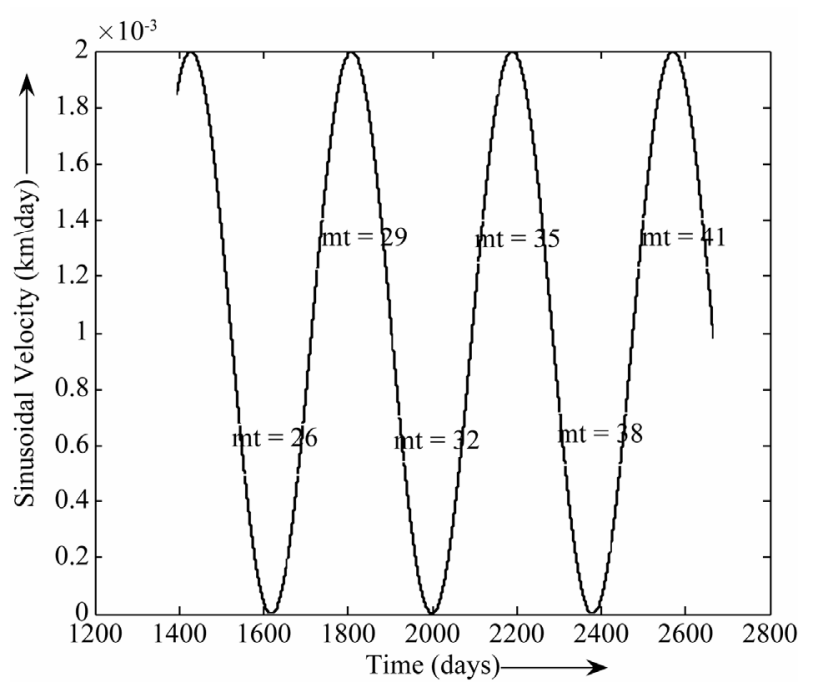

Figure 2. Time dependent sinusoidal form of velocity representations.

Analytical solutions (27) is solved for the values $c_{0}=1.0$, $u_{0}=0.001 \mathrm{~km} / \mathrm{d}, D_{0}=1.0 \mathrm{~km}^{2} / \mathrm{d}$, and $L=100 \mathrm{~km}$. The concentration values in the presence of source pollution till $t=t_{0}(1500 \mathrm{~d})$ are depicted graphically in the presence of constant source of contaminants at $m t=3 k+2$, where $2 \leq k \leq 7$ which represents minimum and maximum records of groundwater level and velocity during June and December in $2^{\text {nd }}, 3^{\text {rd }}$ and $4^{\text {th }}$ years at the respective time values $t(\mathrm{~d})$. When the source is eliminated the solution is solved at $m t=3 k+2$, where $8 \leq k \leq 13$ which represent the duration of June and December alternatively in the $5^{\text {th }}, 6^{\text {th }}$ and $7^{\text {th }}$ years respectively. The contaminants concentration distribution behaviour along unsteady flow of sinusoidal form of velocity given in (28a) depicted in the Figure 3(a) when $T \leq T_{0}$ and Figure 3(b) when $T>T_{0}$. It is observed that the contaminant concentration decreases with time and distance traveled in presence of source contaminants. While in the absence of source contaminants, it increases and goes on increasing which attains towards maximum and then starts decreases and goes on decreasing which attains towards minimum or harmless concentration. This decreasing tendency of contaminant concentration with time and distance traveled may help to rehabilitate the contaminated aquifer. For the same set of inputs except $m=0.0002(/ \mathrm{d})$ as $m t<1, \mathbf{E q . 2 7}$ is also computed for exponentially decreasing form of velocity given in (28b). It is observed that the contaminant concentration follows almost the same trend in presence and absence of source contaminants respectively. This decreasing tendency of contaminant concentration with time and distance traveled is depicted graphically in Figure 4(a) when $T \leq T_{0}$ and Figure 4(b) if $T>T_{0}$ for exponentially decreasing form of velocity.

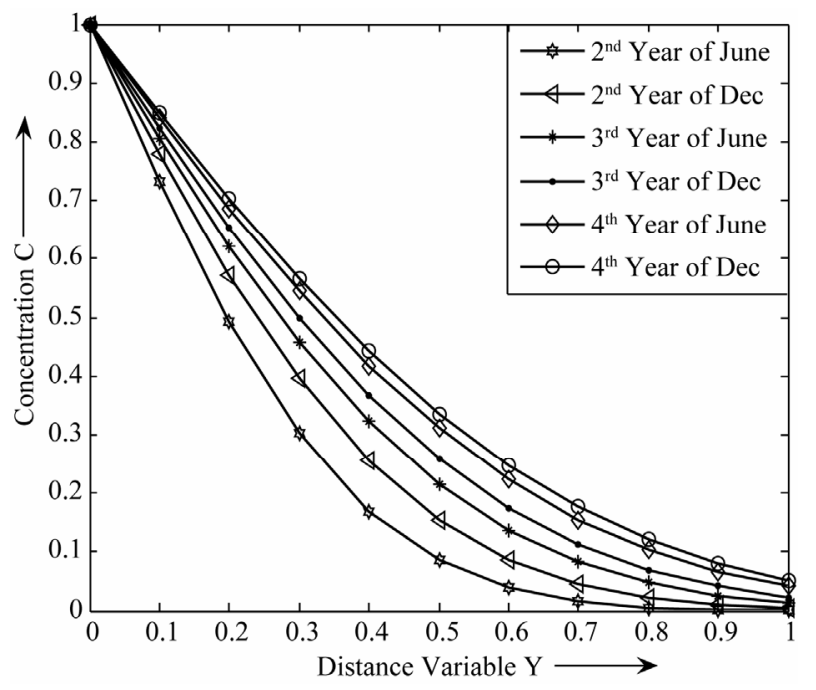

(a)

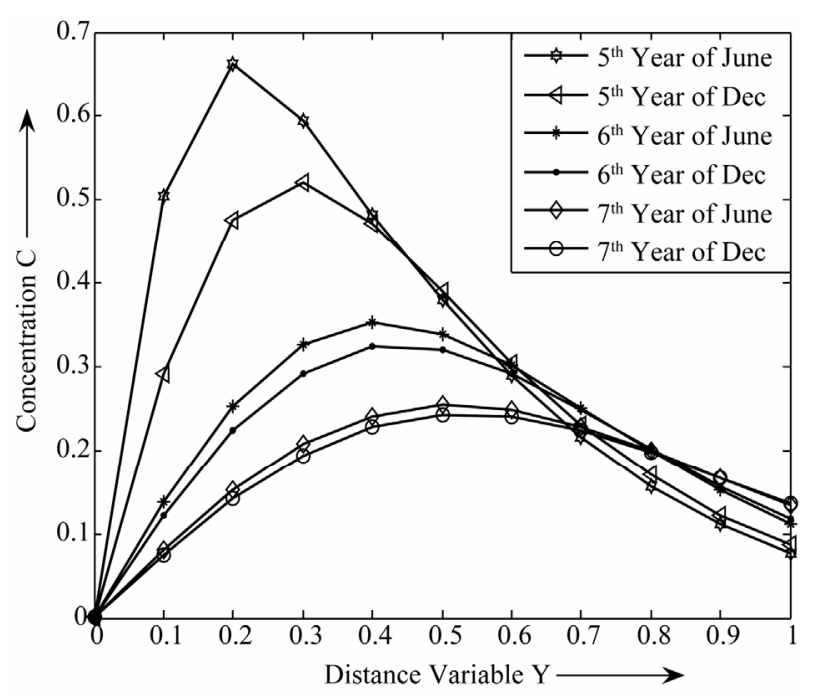

(b)

Figure 3. Contaminants concentration along unsteady groundwater flow of sinusoidal form of velocity in homogeneous finite aquifer when (a) $T \leq T_{0}$ and (b) $T>T_{0}$.

\section{CONCLUSIONS}

A solute transport model is solved analytically with constant source of input concentration in homogeneous finite aquifer. The pulse type boundary conditions are considered in intermediate portion of the aquifer system. The time varying velocities are taken in to consideration in which one such form i.e. sinusoidal form represents the seasonal variation in a year in tropical regions. The Laplace Transform Technique is used to get an analytical solution which is perhaps most useful for benchmarking the numerical codes and models. The result of the problem may be used as the preliminary predictive tools for groundwater management. The solution is obtained and 


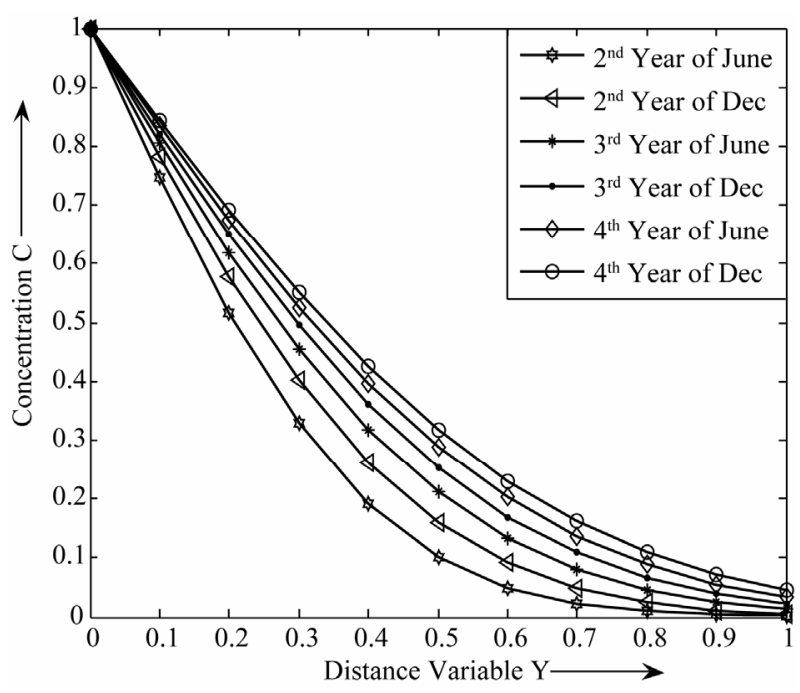

(a)

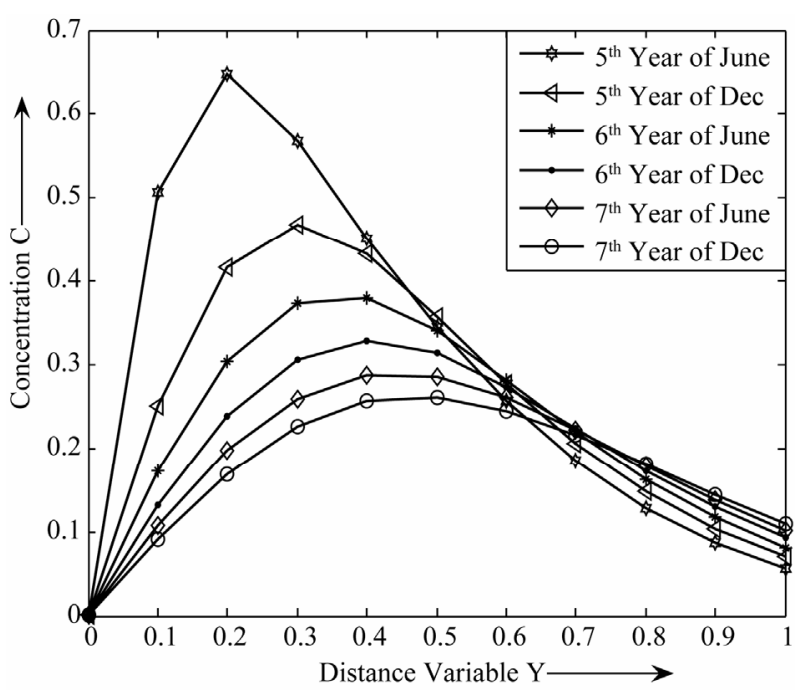

(b)

Figure 4. Contaminants concentration along unsteady groundwater flow of exponential form of velocity in homogeneous finite aquifer when (a) $T \leq T_{0}$ and (b) $T>T_{0}$.

graphical representations are made under the assumption $x \geq x_{0}$ which is the limitation of the solution of the problem. The solution in the domain $x<x_{0}$ is not considered in the present work only because the solute concentration will not remain in this domain for the longer period. After very short duration of time it will move on in the domain $x \geq x_{0}$ [14].

\section{ACKNOWLEDGEMENTS}

The first author is grateful to the University Grants Commission, New Delhi, and Government of India for their financial support to carry out the research work. The authors are thankful to the reviewers for their constructive comments.

\section{REFERENCES}

[1] Fried, J.J. (1975) Groundwater pollution. Elsevier Scientific Publishing Company, Amsterdam.

[2] Ghosh Bobba, A. and Singh, V.P. (1995) Groundwater contamination modeling. In: Singh, V.P., Ed., Enviormental hydrology, Kluwer Academic Publishers, Dordrecht, 225-319.

[3] Charbeneau, R.J. (2000) Groundwater hydraulics and pollutant transport. Prentice Hall, New Jersey.

[4] Rai, S.N. (2004) Role of mathematical modeling. In: Rai, S. N., Ed., Groundwater Resource Management, NGRI, Hyderabad.

[5] Sharma, H.D. and Reddy, K.R. (2004) Geo-environmental Engineering. John Willy \& Sons, INC.

[6] Rausch, R., Schafer, W., Therrien, R. and Wagner, C. (2005) Solute transport modelling: An introduction to models and solution strategies. Gebr. Borntrager Verlagsbuchhandlung Science Publishers, Berlin.

[7] Thangarajan, M. (2006) Groundwater: Resource evaluation, augmnetation, contamination, restoration, modeling and management. Capital Publishing Company, New Delhi, 362.

[8] Gershon, N.D. and Nir, A. (1969) Effects of boundary conditions of models on tracer distribution in flow through porous mediums. Water Resources Research, 5, 830-839. doi:10.1029/WR005i004p00830

[9] Gelher, L.W. and Collins, M.A. (1971) General analysis of longitudinal dispersion in non-uniform flow. Water Resources Research, 7, 1511-1521. doi:10.1029/WR007i006p01511

[10] Marino, M.A. (1974) Longitudinal dispersion in saturated porous media. Journal of the Hydraulics Division, 100, 151-157.

[11] Hunt, B. (1978) Dispersive sources in uniform ground water flow. Journal of the Hydraulics Division, 104, 75-85.

[12] Van Genuchten, M.Th. (1981) Analytical solutions for chemical transport with simultaneous adsorption, zero order production and first order decay. Journal of $\mathrm{Hy}$ drology, 49, 213-233.

[13] Kumar, N. (1983) Unsteady flow against dispersion in finite porous media. Journal of Hydrology, 63, 345-358. doi:10.1016/0022-1694(83)90050-1

[14] Kumar, N. and Kumar, M. (1998) Solute dispersion along unsteady groundwater flow in a semi-infinite aquifer; Hydrology and Earth System Sciences, 2, 93-100. doi:10.5194/hess-2-93-1998

[15] Lindstrom, F.T. and Boersma, L. (1989) Analytical solutions for convective-dispersive transport in confined aquifers with different initial and boundary conditions. $\mathrm{Wa}$ ter Resources Research, 25, 241-256. doi:10.1029/WR025i002p00241

[16] Yates, S.R. (1990) An analytical solution for one-dimensional transport in heterogeneous porous media. $\mathrm{Wa}$ ter Resources Research, 26, 2331-2338. doi:10.1029/WR026i010p02331

[17] Fry, V.A. and Istok, J.D. (1993) An analytical solution to the solute transport equation with rate-limited desorption and decay. Water Resources Research, 29, 3201-3208. doi:10.1029/93WR01394 
[18] Sim, Y. and Chrysikopoulos, C.V. (1995) Analytical models for one-dimensional virus transport in saturated porous media. Water Resources Research, 31, 14291437. doi:10.1029/95WR00199

[19] Chrysikopoulos, C.V. and Sim, Y. (1996) One-dimensional virus transport in homogeneous porous media with time-dependent distribution coefficient. Journal of $\mathrm{Hy}$ drology, 185, 199-219.

[20] Rai, S.N. and Manglik, A. (1999) Modelling of water table variation in response to time varying recharge from multiple basin using the linearised Boussinesq equation. Journal of Hydrology, 220, 141-148. doi:10.1016/S0022-1694(99)00074-8

[21] Sander, G.C. and Braddock, R.D. (2005) Analytical solutions to the transient, unsaturated transport of water and contaminants through horizontal porous media. Advances in Water Resources, 28, 1102-1111. doi:10.1016/j.advwatres.2004.10.010

[22] Srinivasan, V., and Clement, T.P. (2008) Analytical solutions for sequentially coupled one-dimensional reactive transport problems-Part-I: Mathematical Derivations. Advances in Water Resources, 31, 203. doi:10.1016/j.advwatres.2007.08.002

[23] Singh, M.K., Mahato, N.K. and Singh, P. (2008) Longitudinal dispersion with time dependent source concentra- tion in semi infinite aquifer. Journal of Earth System Science, 117, 945-949.

doi:10.1007/s12040-008-0079-x

[24] Singh, M.K., Singh, V.P., Singh, P., and Shukla, D. (2009) Analytical solution for conservative solute transport in one-dimensional homogeneous porous formations with time dependent velocity. Journal of Engineering $\mathrm{Me}$ chanics, 135, 1015-1021. doi:10.1061/(ASCE)EM.1943-7889.0000018

[25] Singh, M.K., Singh, P. and Singh, V.P. (2010) Two dimensional solute dispersion with time dependent source concentration in finite aquifer. Journal of Engineering Mechanics, 136, 1309-1315. doi:10.1061/(ASCE)EM.1943-7889.0000177

[26] Ebach, E.H. and White, R. (1958) Mixing of fluid flowing through beds of packed solids. Journal of American Institute of Chemical Engineers, 4, 161-164.

[27] Rumer, R.R. (1962) Longitudinal dispersion in steady and unsteady flow. Journal of the Hydraulics Division, 88, 147-172.

[28] Crank, J. (1975) The Mathematics of diffusion. Oxford University Press, Oxford.

[29] Banks, R.B. and Jerasate, S. (1962) Dispersion in unsteady porous media flow. Journal of the Hydraulics Division, 88, 1-21. 